\title{
Comparing morphology, flow cytometry and molecular genetics in the assessment of minimal residual disease in children with $B$-acute lymphoblastic leukaemia (B-ALL)
}

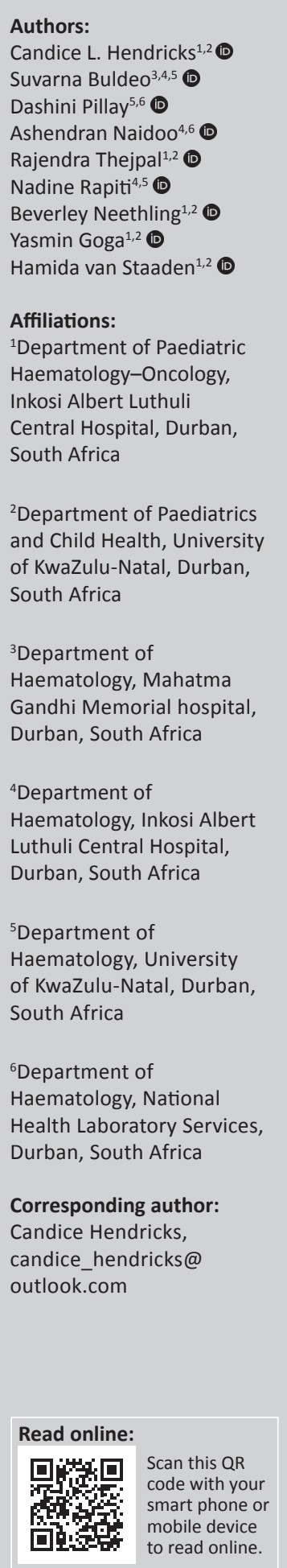

Background: Minimal residual disease (MRD) detection has been shown to be the best prognostic factor in B-acute lymphoblastic leukaemia (B-ALL). Multicolour flow cytometry (FCM) and specific molecular aberrations (MOL) are the classic techniques used to assess MRD. The former is faster and less costly.

Aim: This study compares morphology and FCM to MOL in detecting MRD.

Setting: The study was conducted at Inkosi Albert Luthuli Central Hospital (IALCH).

Methods: A retrospective review of children with B-ALL managed at IALCH from January 2013 to January 2018 was conducted. Multicolour flow cytometry was performed using Euroflow ${ }^{\circledR}$ panels. Molecular aberrations looked at common cytogenetic markers. Presentation and post-induction morphology (May-Grunwald Giemsa stain), FCM and MOL data for MRD were analysed.

Results: Eleven patients were excluded (6-demised, 5-incomplete records), leaving 64 to be analysed ( $54 \%$ female, median age 5 years). Five post-induction aspirates were unsuitable but the rest (92\%) were in morphological remission. At diagnosis and post-induction, 62 (95\%) and $61(94 \%)$ children, respectively, had FCM performed. A positive MOL result was found in 39 $(60 \%)$ patients. MOL turn-around times (TATs) averaged 14 days compared with those of FCM's average of 3 days. MRD was found in 9 patients (FCM) and 7 patients (MOL). Of these patients, 4 had a good correlation between the two and 2 patients with negative FCM had positive MOL MRD post-induction.

Conclusion: Morphology is insensitive in MRD assessment. FCM correlated well with molecular MRD and has the shortest turn-around time. FCM has major benefit in the $40 \%$ of patients with negative MOL. It can also be safely used to guide treatment escalation in those patients awaiting molecular results.

Keywords: minimal residual disease; B-acute lymphoblastic leukaemia; paediatric; flow cytometry; molecular genetics.

\section{Introduction}

The survival of children diagnosed with B-acute lymphoblastic leukaemia (B-ALL) has increased markedly, reaching $>80 \%$ in developed countries. ${ }^{1,2,3}$ These outcomes are largely attributed to better risk stratification of patients, allowing therapy to be tailored accordingly. ${ }^{2}$ Risk categories take into account age of the patient, white cell count at diagnosis, recurrent genetic abnormalities and treatment response. ${ }^{1,4}$ Early response to treatment can be assessed by morphology or flow cytometry (FCM). Morphology is usually assessed by a peripheral blood blast count on Day 8 and by an FCM at Day 8 and Day 33 (post-induction chemotherapy). ${ }^{5}$ The Day 33 sample is a bone marrow aspirate sample. The latter plays a major role in stratifying these patients, even in the absence of other poor risk factors. ${ }^{5}$

The detection of minimal residual disease (MRD), more aptly referred to currently as measurable residual disease, early on in treatment is one measure used to identify patients at high risk of

Dates: Received: 11 Mar. 2019 | Accepted: 14 Aug. 2019 | Published: 23 Oct. 2019

How to cite this article: Hendricks CL, Buldeo S, Pillay D, et al. Comparing morphology, flow cytometry and molecular genetics in the assessment of minimal residual disease in children with B-acute lymphoblastic leukaemia (B-ALL). S. Afr. j. oncol. 2019;3(0), a76. https:// doi.org/10.4102/sajo.v3i0.76

Copyright: @ 2019. The Authors. Licensee: AOSIS. This work is licensed under the Creative Commons Attribution License. 
treatment failure or relapse. It has also been shown to be the most important factor determining the outcome of patients undergoing a bone marrow stem cell transplant. ${ }^{6}$ MRD is defined as the detection of greater than 1 leukaemia cell in 10000 normal cells $(>0.01 \%)^{7}$ and has been shown to be the best prognostic factor in patients with B-ALL. ${ }^{7}$ MRD negativity generally predicts a very low risk of relapse and the ability to de-escalate management. ${ }^{4}$ The three classic MRD techniques are multicolour FCM (to detect a leukaemia-associated immunophenotype [LAIP]), monoclonal immunoglobulin gene rearrangements by real-time quantitative polymerase chain reaction (PCR) and the detection of fusion genes by PCR. ${ }^{8,9}$ MRD by FCM relies on the detection of a LAIP at diagnosis, distinguishing the abnormal population of blasts by over-, under- or aberrant antigen expression. . $^{10,11,12}$

PCR methods are more sensitive than standard immunophenotyping ${ }^{13}$ but FCM is known to be quicker, less costly and less likely to pick up apoptotic blast cells as compared to the molecular methods..$^{1,5,14}$ There is however the possibility of therapy changing thepatient'simmunophenotype leading to the recommendation that at least two antigenic markers be used to define the LAIP per patient. ${ }^{5}$

It is important to note that although FCM and molecular techniques have theirindividual advantages and disadvantages, both methods show good correlation in MRD detection. ${ }^{1}$ Minimal residual disease, however should not be interpreted in isolation as the combination of MRD with poor clinical risk factors bears the worst outcomes for patients. ${ }^{14}$

Morphological assessment of bone marrow aspirates post induction is performed in most cancer centres to assess the remission status. ${ }^{15}$ In the late 1990s when MRD was gaining popularity in risk stratification, it was noted that the presence of blasts on morphology was a poor marker for risk of relapse, as it was not possible to distinguish pathological blasts from normal haematogones. ${ }^{16}$ Because of its decreased sensitivity compared to FCM and MOL methods, its value in determining remission status is waning. ${ }^{17}$ With a rise in the burden of noncommunicable diseases in South Africa ${ }^{18}$ and the cost of treating malignancies known to be significant, ${ }^{19}$ omitting a test that has limited benefit in patient management is inevitable.

Inkosi Albert Luthuli Central Hospital (IALCH) is a quaternary hospital in Durban, South Africa, whose Haematology-Oncology Unit serves all children with malignancies from throughout KwaZulu-Natal. The IALCH unit has 29 beds catering for these children with access to both high care and ICU facilities on site.

The haematology laboratory provides in-house morphology and FCM services. MRD by FCM has been used in the laboratory since 2013 and is now used as a measure to guide molecular testing as well as clinical management. Because of unforeseen technical problems, FCM is sometimes referred to other sites. In patients with B-ALL, FCM for MRD is performed at Day 33 (post induction) as the initial measure of MRD. Limited molecular services are provided with most tests being outsourced to either the private sector or other state laboratories.

In this study, children diagnosed with B-ALL were reviewed to compare the diagnostic, as well as the post-induction morphology, FCM and molecular testing, results. The aim was to determine a testing algorithm which can guide patient management to ensure the most justifiable, sustainable and optimal use of hospital resources at a reasonable cost without compromising patient care.

\section{Materials and methods Study design and study population}

A retrospective review of patient records was conducted using the electronic database of the Haematology-Oncology Unit to identify patients aged 0-13 years with a diagnosis of B-ALL from 01 January 2013 to 31 January 2018. Children older than 13 years and those who demised before post-induction chemotherapy investigations were excluded. The data were anonymous, password protected and only accessible to the primary investigator. The hospital and laboratory information systems were searched to retrieve the required clinical and laboratory information. Ethical approval was obtained from the Biomedical Research Ethics Committee at the University of KwaZulu-Natal. Permission was obtained from the laboratory and hospital management to collect these results.

The laboratory techniques for diagnosing and monitoring of residual leukaemic disease post-induction chemotherapy included morphology assessment, FCM and molecular genetic analysis.

\section{Morphology}

Bone marrow aspirate slides were stained with the May-Grunwald Giemsa stain and reviewed by a registrar, and findings were corroborated by a pathologist under a Leica light microscope (Leica Microsystems, Germany). A myelogram count was performed on 500 nucleated cells to quantify the number of blasts using the 50× magnification, and this was documented on the bone marrow aspirate report that was retrieved from the laboratory information system.

\section{Flow cytometry}

Flow cytometry (FCM) monitoring followed the adapted Euroflow protocols ${ }^{20,21}$ as per the laboratory standard operating procedures and acquired on the Becton, Dickinson and Company (BD) Facscanto II FCM (San Jose, CA, USA). Samples were collected into ethylenediamine tetraacetic acid (EDTA) collection tubes and were processed within 48 hours of collection. The choice of markers selected for MRD monitoring was dependent on antigenic aberrancies that existed on the presentation sample. The following markers were most commonly used: CD20, CD45, CD58, CD66c, CD34, CD19, CD10, CD38, CD21, Cd15, NG2, CD123 and CD81. The flow cytometry data files were analysed with the Infinicyt software, v1.7 (Cytognos SL Salamanca, Spain), 
and Infinicyt reference images of the presentation immunophenotype were used to guide MRD analysis. Results were interpreted by a pathologist, and the report was uploaded to the laboratory information system.

\section{Molecular analysis}

Molecular testing (MOL) analyses included studies for $t(1 ; 19), t(9 ; 22) \mathrm{p} 190, t(12 ; 21)$, MLL gene rearrangement and immunoglobulin heavy chain gene rearrangements. Peripheral blood or bone marrow aspirate samples were analysed by quantitative real-time PCR (ipsogen, Qiagen, Germany or Identiclone, InVivoScribe, USA [detection limit of 1 in 10000 cells]) or fluorescent in situ hybridisation (FISH) (VYSIS FISH probes, Abbot, USA [detection limit of 1 in 200 interphase cells]) to identify the genetic aberrations. A mix of forward and reverse primers was used as per the Ipsogen kits. Standard operating protocols were used, and results were retrieved from the laboratory information systems.

The detection of residual disease between the different laboratory techniques was compared.

\section{Turn-around time}

Turn-around time (TAT) was defined as the time from when the sample was received in the laboratory to the time at which the result was accessible on the laboratory (Labtrack) information system. The TATs for morphology, FCM and molecular techniques were compared to establish which method yielded a result within the shortest amount of time.

\section{Statistical analysis}

Descriptive statistics was used to summarise the data. Frequencies and percentages were used for categorical data. Diagnostic test measures such as sensitivity and specificity, positive predictive value and negative predictive value were used to compare FCM with the gold standard test, that is, MOL. Flow cytometry and MOL testing were correlated with Cohen's kappa and Pearson's correlation coefficient.

\section{Ethical considerations}

Biomedical Research Ethics committee of the University of KwaZulu-Natal provided ethical clearance for this study, BREC Reference number: BE203/18. Permission to conduct the study was granted by the Inkosi Albert Luthuli Central Hospital (IALCH) upon approval received from the KwaZulu-Natal Department of Health, Health research and knowledge management (HRLM), Ref: 254/18 and National Health research database (NHRD), Ref: KZ_201806_035.

\section{Results}

Seventy-five patients were diagnosed with B-ALL during the study period. Six demised during the induction period and five patients had incomplete records. Sixty-four patients (Figure 1) were included in the study, and at diagnosis morphological assessment, FCM and MOL testing were performed in $81 \%, 97 \%$ and $100 \%$, respectively, of all the study participants.

The demographic characteristics of the study population are summarised in Table 1. Females made up 54.6\%, and the median age of this patient group was 5 years. Thirty-five patients $(54.6 \%)$ were under the age of 5 years, and only one patient $(1.6 \%)$ was older than 10 years.

Figure 2 represents the MRD results of the study population. MRD was detected in 12 patients. Five aspirates were unsuitable (hypocellular/dilute), while the remaining 92\%

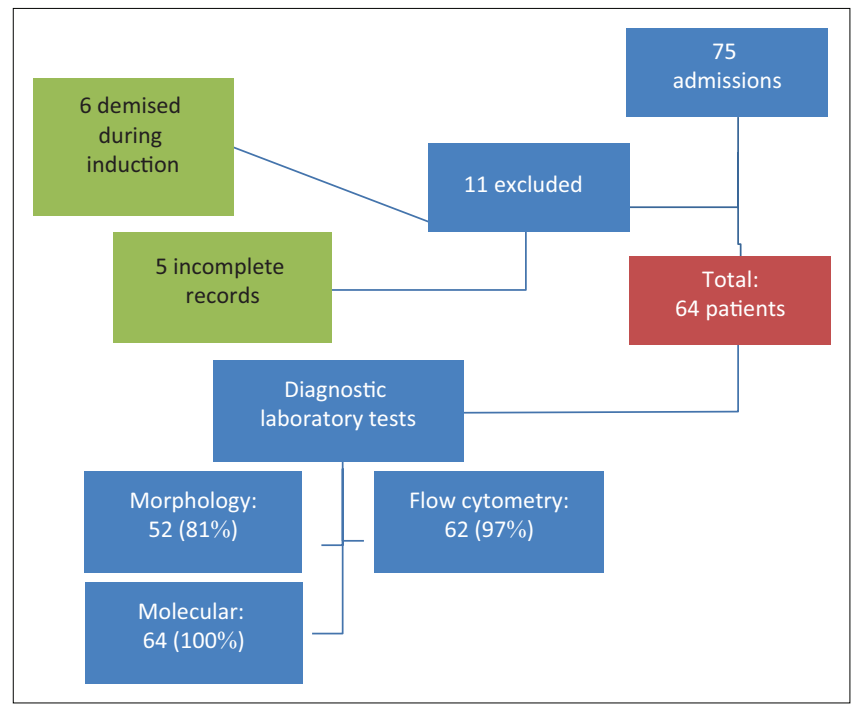

FIGURE 1: Patient population.

TABLE 1: Demographic characteristics.

\begin{tabular}{lcc}
\hline Variable & Number & Percentage of total \\
\hline Gender & 29 & \\
Male & 35 & 45.3 \\
Female & & 54.6 \\
Age & 35 & \\
$1-<5$ years & 28 & 54.6 \\
$5-<10$ years & 1 & 43.8 \\
$10-<13$ years & & 1.6 \\
\hline
\end{tabular}

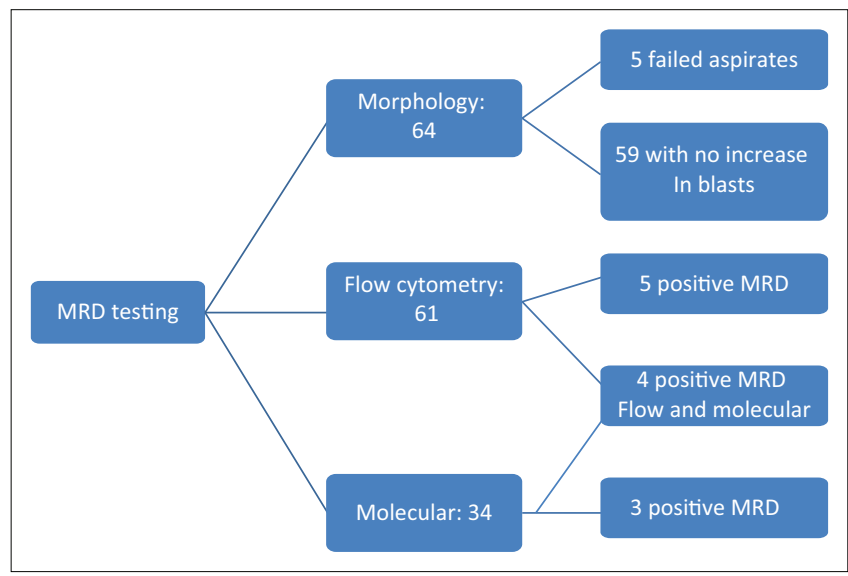

MRD, Minimal residual disease.

FIGURE 2: Post-induction minimal residual disease results. Minimal residual disease was detected in 12 patients, 9 by flow cytometric methods and 7 by molecular techniques. 
showed morphological remission ( $<5 \%$ blasts seen). A positive MOL result was found in 39/64 (60\%) patients at diagnosis, and in 34 patients the assay was repeated post induction. Flow MRD was performed in 61 of the 64 patients. The remaining three patients were from 2013 when FCM was not yet routinely used as part of MRD protocols in the unit.

Flow cytometry identified an MRD population in 9/61 (15\%), while MOL testing identified MRD in 7/64 (11\%). If only including those patients in whom a positive molecular result was found at diagnosis, this figure increases to $18 \%$. FCM and MOL MRD results correlated for four patients. Of the remaining 5 flow MRD positive results, two patients did not have a positive molecular result at diagnosis and one patient had a monoclonal immunoglobulin heavy chain gene rearrangement (monoclonal $\mathrm{IgH}$ ) at diagnosis which was negative post induction. The remaining two patients were hyperdiploid at diagnosis without other positive molecular results, and ploidy analysis was not repeated post induction (threshold of blast population not present for ploidy analysis). Two of the seven patients with a positive MOL MRD had negative FCM. One patient had a monoclonal $\operatorname{IgH}$, and the other had a $t(1 ; 19)$ fusion transcript. One patient had MOL MRD positive (fusion transcript) but MRD FCM was not performed in this patient. Using MOL as the gold standard, the positive and negative predictive values of FCM were found to be $80 \%$ and $92 \%$, respectively. The sensitivity of FCM in our cohort was $66 \%$, while the specificity was $96 \%$. The correlation coefficient between the two tests was 0.71 using Pearson's correlation coefficient and 0.66 using Cohen's kappa.

Figure 3a-g shows the residual disease detected by FCM including one patient with antigenic shifts (Figure 3f). These patients had MRD testing performed at IALCH. The patient in Figure $3 e$ had a diagnostic FISH for $t(1 ; 19)$ that was negative. In view of the positive MRD finding and the LAIP favouring a

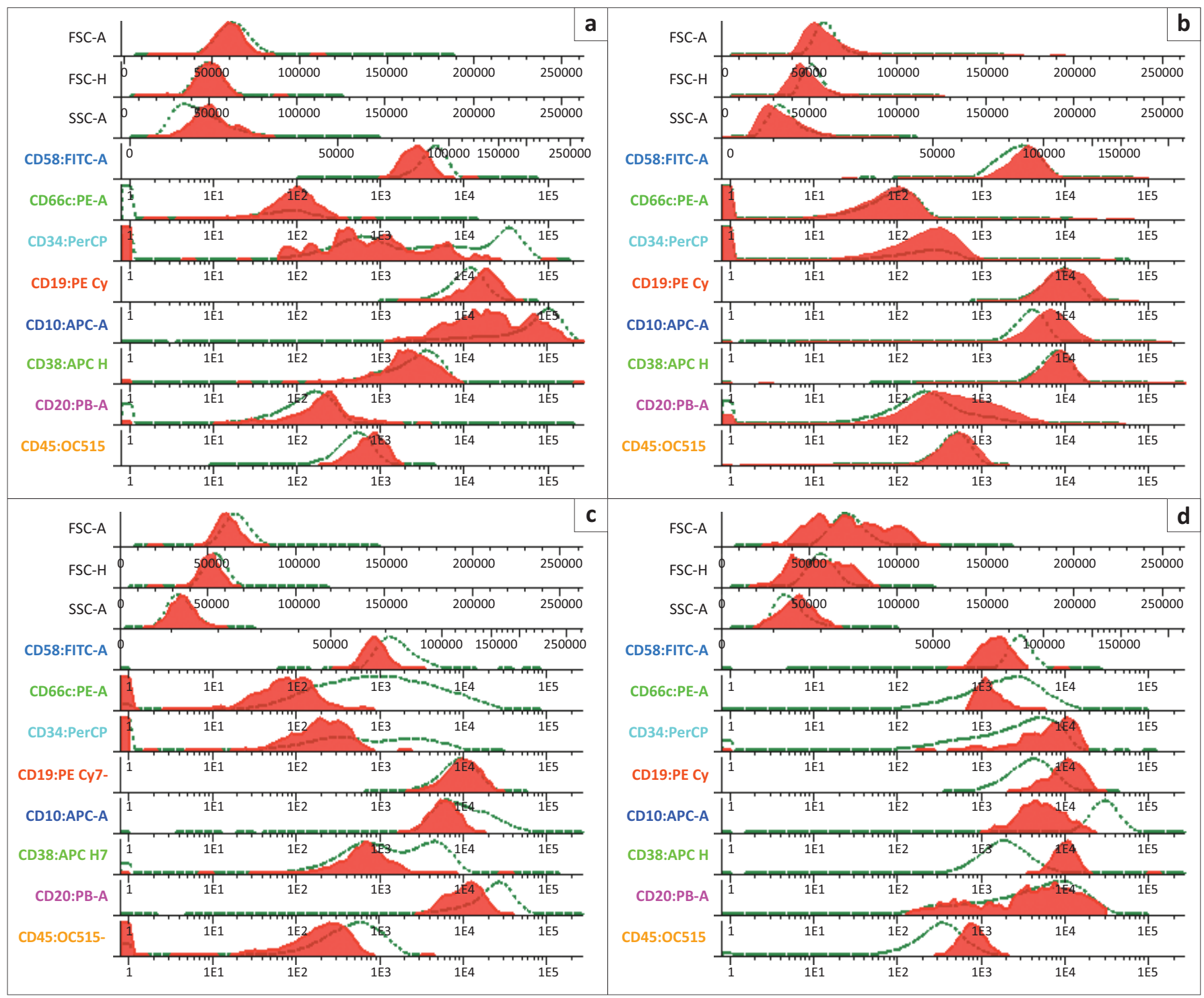

MRD, Minimal residual disease.

FIGURE 3: Histograms illustrating patients with flow minimal residual disease positivity. (a) flow MRD pos: $t(12 ; 21)$ pos; (b) flow MRD pos: $t(1 ; 19)$ pos; (c) flow MRD pos: hyperdiploidy; (d) flow MRD pos: hyperdiploidy; (e) flow MRD pos: $t(1 ; 19)$ pos; (f) flow MRD pos: hyperdiploidy with antigenic shifts; (g) flow MRD positive (monoclonal IgH). 


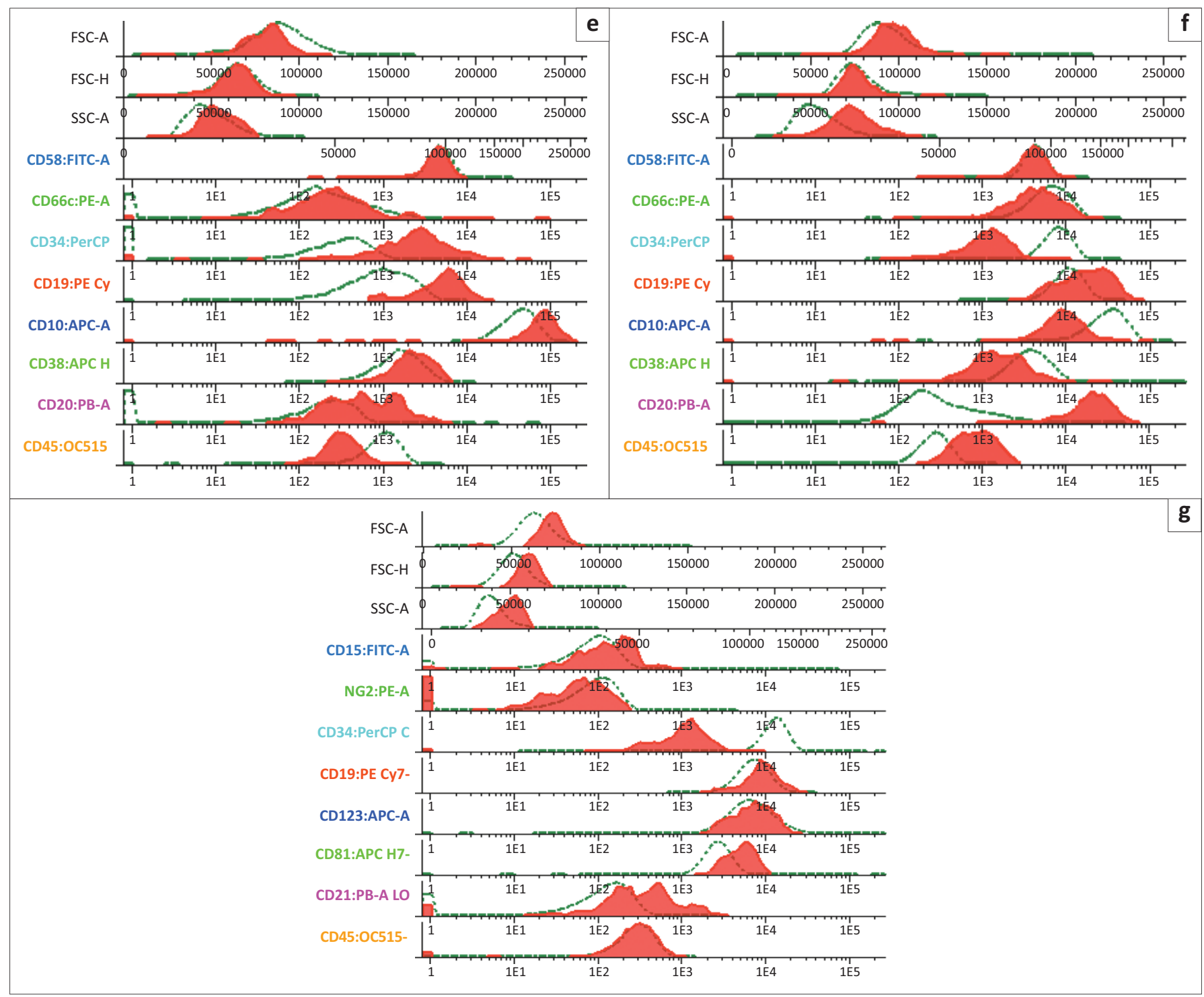

MRD, Minimal residual disease.

FIGURE 3 (Continues...): Histograms illustrating patients with flow minimal residual disease positivity. (a) flow MRD pos: $t(12 ; 21)$ pos; (b) flow MRD pos: $t(1 ; 19)$ pos; (c) flow MRD pos: hyperdiploidy; (d) flow MRD pos: hyperdiploidy; (e) flow MRD pos: $t(1 ; 19)$ pos; (f) flow MRD pos: hyperdiploidy with antigenic shifts; (g) flow MRD positive (monoclonal IgH).

$t(1 ; 19)$, a PCR was repeated post induction and found to be positive. The LAIP for each patient is shown by the green reference line, and the post-induction MRD assessment is shown by the red histograms. All MRD assessments are also referenced to haematogones (not shown).

Figure 4 portrays the TATs of the different laboratory tests at diagnosis and post induction. Flow cytometry was superior with an average TAT of 3 days overall. Molecular results took an average of 15 and 13 days at diagnosis and post induction, respectively.

One patient with positive MRD defaulted but most remaining patients had treatment intensification. These results are shown in Figure 5. Eight patients (67\%) received the Berlin-FrankfurtMunich (BFM) 95 high-risk chemotherapy blocks, ${ }^{22}$ one patient had induction therapy extended for 2 weeks and another had re-induction therapy. One patient remained on the standard chemotherapy protocol possibly because molecular results

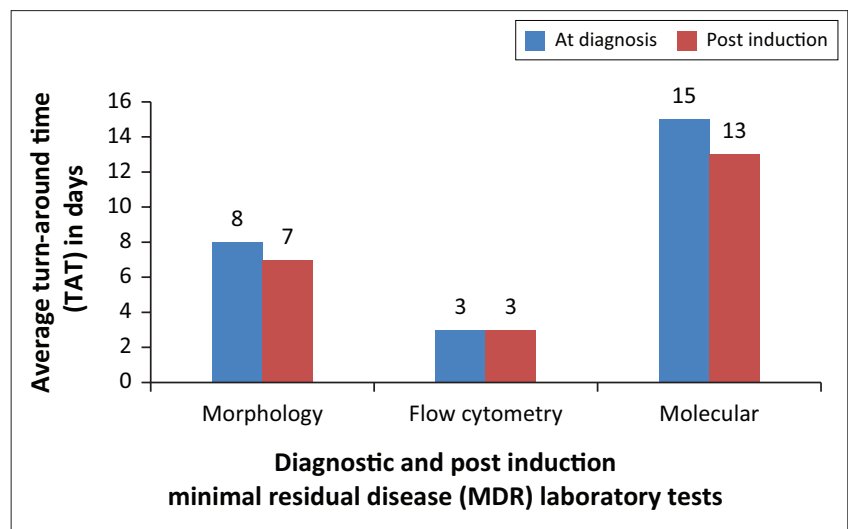

FIGURE 4: Comparison of turn-around times.

were not available yet at the time that a decision regarding treatment intensification had to be made. Six of the MRD positive patients $(50 \%)$ demised, three from relapse, one from refractory disease (patient defaulted and demised at base 


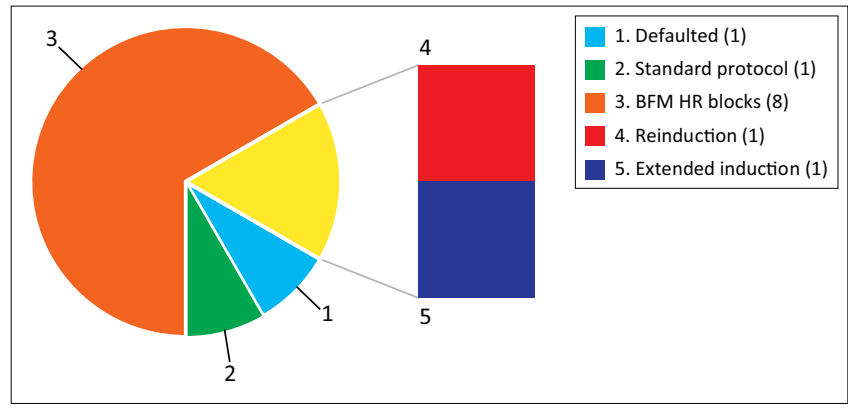

BFM HR, Berlin -Frankfurt -Munich high-risk.

FIGURE 5: Clinical decision based on positive minimal residual disease (\%).

hospital) and two from sepsis. This is higher compared to the MRD negative population in whom the mortality rate was $12 \%(6 / 52)$ with five of these patients relapsing.

\section{Discussion}

The detection of minimal residual disease (MRD) in haematological malignancies has provided a means of better risk stratification of patients. ${ }^{11,23,24}$ Patients who are shown to have MRD at the end of induction therapy are at a higher risk of relapse (as shown in this study) and have been shown to benefit from treatment intensification. $8,23,25$ Furthermore, studies have shown that patients without MRD can have treatment deescalation without increased risk of morbidity or mortality. ${ }^{26,27,28}$ The impact of MRD on patient management has led to the need for standardised practices to be implemented, culminating in the recent publication of the AIEOP-BFM Consensus Guidelines 2016 for Flow Cytometric Immunophenotyping of Paediatric Acute Lymphoblastic Leukaemia. ${ }^{29}$

In this study, $15 \%$ of patients were found to have MRD by FCM, and $18 \%$ of patients were found to have MRD by molecular methods (according to the number of patients that had a positive molecular result at diagnosis). In a large Children's Oncology Group study, 19\% of patients were found to have MRD ${ }^{30}$; however, other studies show higher numbers, that is, $25-28 \%$ of patients had MRD post induction. ${ }^{31,32}$ The lower MRD rate in our population may be related to the small sample size and the fact that the flow panel and the instrument used in the two studies were different from those used in this study. Decisions to escalate therapy were made based on MRD results as well as any additional high-risk features that patients may have presented with. The majority of patients $(85 \%)$ received intensified chemotherapy. Currently, MRD by FCM is the standard practice in the United States, while Europe uses q-PCR. ${ }^{33}$

The correlation between FCM and MOL MRD detection in this study was found to be 0.66 by Cohen's kappa and 0.71 by Pearson's correlation coefficient. This is lower than that found in the international literature $\mathrm{r}^{8,9,13,34}$ and may be a function of sample size. Discordance between FCM and PCR MRD methods is usually present in patients with low levels of MRD (0.001\%). ${ }^{35}$ This can usually be overcome by next-generation sequencing, which can increase the concordance to $98 \%,,^{13,36}$ as well as reduced by newer processing methods for FCM such as bulk-lysis protocols. Likely reasons for discordance could be a poor sample (too few events, contamination and coagulation), ${ }^{37}$ different laboratories using different techniques for MRD detection and the presence of normal regenerating B-cell precursor cells, particularly in regenerating bone marrow after induction chemotherapy. ${ }^{\text {? }}$

Flow cytometry is known to be a 'faster and less labourintensive' method to detect MRD. ${ }^{7}$ This study concurs with this statement in that the TAT was reduced compared to both morphology and molecular results, with telephonic flow results available within 24 hours. Additionally, the entire bone marrow sample is assessed, and antigenic shifts, whether from clonal evolution or clonal selection, can be detected (Figure 3). Clonal evolution is known to be an important factor in the determination of MRD as treatment can alter the expression of certain antigens leading to falsenegative results. ${ }^{33}$ Flow cytometry can be used to monitor $>90 \%$ of patients (especially in patients with two or more markers to define the LAIP) ${ }^{38}$ for MRD, unlike MOL testing which can only be performed if the genetic aberration was present at diagnosis. Added advantages of FCM are that very small populations of cells can be identified and dead cells can be recognised and excluded from analysis. ${ }^{37}$ Molecular aberrations were only detected in $60 \%$ of the study participants at diagnosis, and hence $40 \%$ could not be followed up using MOL methods. This is higher than that found in other studies where a prevalence of fusion transcripts is found in up to $40 \%$ of patients. ${ }^{7,24,35}$ In our study, FCM results were used to guide patient management in these patients.

The sensitivity of flow cytometry in this study was $66 \%$, and the specificity was $96 \%$. Two patients had a negative flow MRD but a positive MOL MRD. One of these patients had a fusion transcript, and another had a monoclonal immunoglobulin heavy chain product $(\mathrm{IgH})$ which remained positive post induction. In both of these patients, the paired diagnostic and MRD FCM techniques were not performed in the same laboratory, and hence no reference images were available at the time of doing MRD testing at IALCH. This may have been a limitation to MRD detection. Rarely, however, residual disease is not the only reason for monoclonal $\mathrm{IgH}$ detection. Both non-malignant conditions and viral infections must also be considered. ${ }^{39}$

Similar to other studies, morphological analysis of bone marrow aspirate samples was not sensitive in the postinduction detection of MRD. ${ }^{17,25,27}$ This is likely because of induction chemotherapy often resulting in a hypoplastic bone marrow and morphological changes to cells. Furthermore, it is difficult to morphologically differentiate clonal precursor B cells (in a regenerating bone marrow) from haematogones ${ }^{40}$ which could lead to over- or underestimation of disease burden. ${ }^{24}$ The technique and sample quality greatly affect the ability to detect abnormal cells. ${ }^{34} \mathrm{~A}$ recent study from the Children's Oncology Group (COG) compared 
morphology to flow cytometry in the assessment of remission. Morphology was found to be inferior to flow cytometry in the assessment of remission, and hence the authors suggested that flow cytometry should 'augment the definition of remission in ALL'. ${ }^{17}$ The need to include morphology is thus not justified, especially considering the added cost as well as the unnecessary clinical workload on the pathologists.

The limitations of this study are that the sample size was small, and MRD testing was not performed on Day 8 or 15 to compare results. Most molecular tests were referred to other laboratories which impacted on the TAT but this may be applicable to many other centres as molecular labs are not widely available. The standard, rather than bulk lysis, flow cytometry protocols were used; however, the laboratory uses the same method to process proficiency samples and is showing very good performance. The sensitivity of flow cytometry is also improved by the use of case-specific LAIP markers as well as immunophenotypic shifts whether treatment-related or not. ${ }^{37}$

Although a full cost-effectiveness analysis was beyond the scope of this article, there is a clear cost-saving should morphology be excluded from post-induction (and other time-points) analysis for minimal residual disease. The evidence shows that this can be done without compromising patient care or safety. The benefit will also extend to the workload of the two pathologists at the haematology laboratory who would have to ensure the reporting of these morphology cases. The WHO has advocated for the use of a workload indicator tool, the WISN (Workload Indicators of Staffing Need), which assesses workload pressures in health care facilities, ${ }^{41}$ clearly indicating the need to ensure maximum productivity of staff which is greatly aided by excluding unnecessary work.

A possible algorithm for post-induction minimal residual disease monitoring is shown in Figure 6:

- At diagnosis: Collect bone marrow for an aspirate, trephine, flow and molecular testing. Review morphology and process the FCM sample. Based on the LAIP, order the appropriate molecular tests that are most commonly associated with that particular immunophenotype.

- Post induction: Collect bone marrow for aspirate, trephine, flow and molecular testing. Flow cytometry sample should be the first draw, followed by molecular sample. Process flow cytometry sample; if positive treat as MRD. If negative, do MOL tests if present from diagnosis. If MOL not detected, review morphology.

\section{Conclusion}

Morphology is insensitive in MRD assessment and can safely be excluded with the added benefit of decreased pressure on human resources and a significant cost saving. Flow cytometry has a major benefit in $40 \%$ of patients with a negative MOL test at diagnosis and has the shortest TAT. It can also be safely used to guide treatment escalation in those patients awaiting molecular results. Flow cytometry

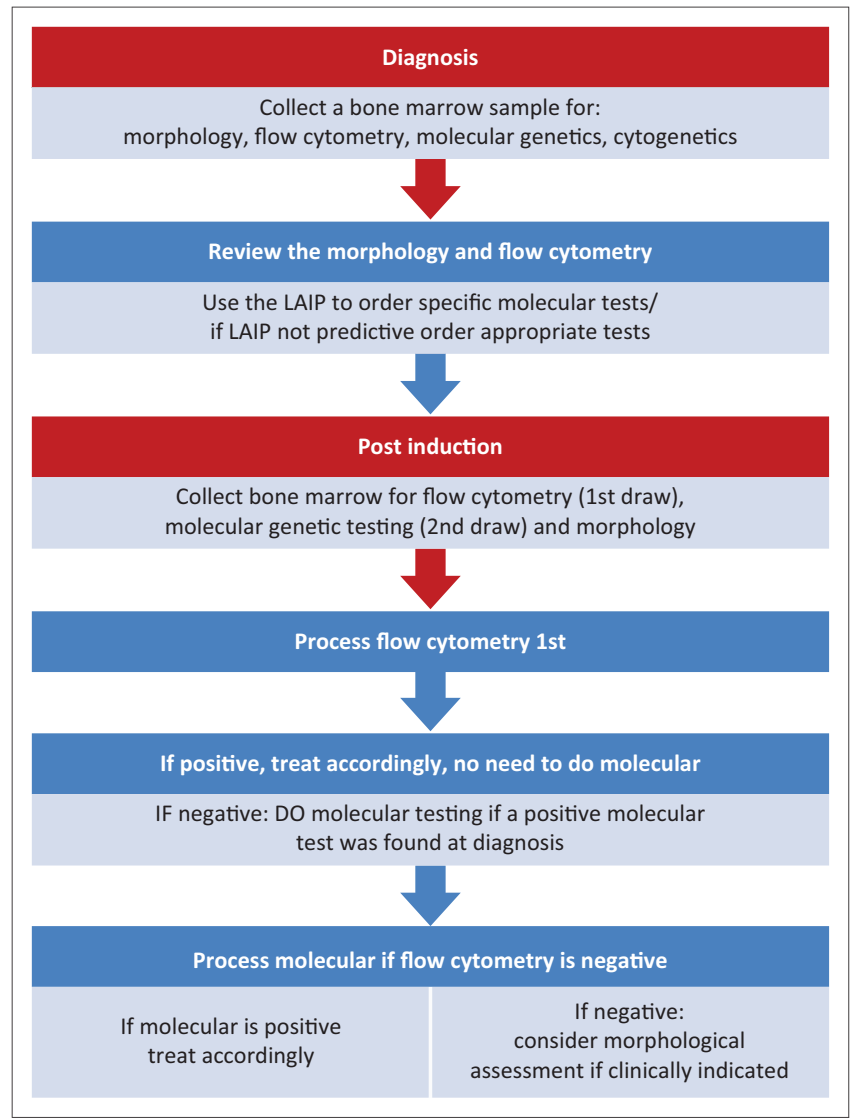

MRD, minimal residual disease.

FIGURE 6: Proposed testing algorithm for minimal residual disease.

diagnostic and follow-up MRD samples should always be reviewed in the same laboratory to ensure that reference images are available. The lack of complete survival data was a limitation of the study.

\section{Acknowledgements Competing interests}

The authors have declared that no competing interest exists.

\section{Authors' contributions}

C.L.H., S.B., D.P. and A.N. were responsible for the conceptualisation of the study, protocol preparation, data analysis and write-up of the manuscript. R.T. and N.R. were responsible for the data analysis and write-up of the manuscript. B.N., Y.G. and H.v.S. were responsible for the data collection, data analysis and write-up of the manuscript.

\section{Funding}

This research received no specific grant from any funding agency in the public, commercial or not-for-profit sectors.

\section{Data availability statement}

New data were created and analysed in this study. Authors may be contacted in this regard. 


\section{Disclaimer}

The views and opinions expressed in this article are those of the authors and do not necessarily reflect the official policy or position of any affiliated agency of the authors.

\section{References}

1. Irving J, Jesson J, Virgo P, et al. Establishment and validation of a standard protoco for the detection of minimal residual disease in $B$ lineage childhood acute lymphoblastic leukemia by flow cytometry in a multi-center setting. Haematologica. 2009;94(6):870-874. https://doi.org/10.3324/haematol.2008.000414

2. Yeoh AEJ, Ariffin H, Chai ELL, et al. Minimal residual disease-guided treatment deintensification for children with acute lymphoblastic leukemia: Results from the Malaysia-Singapore acute Iymphoblastic leukemia 2003 study. J Clin Oncol. 2012;30(19):2384-2392. https://doi.org/10.1200/JCO.2011.40.5936

3. Pui $\mathrm{CH}$, Evans WE. A 50-year journey to cure childhood acute lymphoblastic leukemia. Semin Haematol. 2014;50(3):185-196. https://doi.org/10.1053/j. seminhematol.2013.06.007

4. Dworzak MN, Fröschl G, Printz D, et al. Prognostic significance and modalities of flow cytometric minimal residual disease detection in childhood acute lymphoblastic leukemia. Blood. 2002;99(6):1952-1958. https://doi.org/10.1182/ blood.v99.6.1952

5. Ratei R, Basso G, Dworzak M, et al. Monitoring treatment response of childhood precursor B-cell acute lymphoblastic leukemia in the AIEOP-BFM-ALL 2000 protocol with multiparameter flow cytometry: Predictive impact of early blast reduction on the remission status after induction. Leukemia. 2009;23(3):528-534. https://doi.org/10.1038/leu.2008.324

6. Zhao X-S, Liu Y-R, Zhu H-H, et al. Monitoring MRD with flow cytometry: An effective method to predict relapse for ALL patients after allogeneic hematopoietic stem cell transplantation. Ann Hematol [Internet]. 2012;91(2):183-192. ***Available from: http://link.springer.com/10.1007/s00277-011-1285-1.

7. Van Dongen JJM, Van Der Velden VHJ, Brüggemann M, Orfao A. Minimal residual disease diagnostics in acute lymphoblastic leukemia: Need for sensitive, fast, and standardized technologies. Blood. 2015;125(26):3996-4009. https://doi.org/ 10.1182/blood-2015-03-580027

8. Thörn I, Forestier E, Botling J, et al. Minimal residual disease assessment in childhood acute lymphoblastic leukaemia: A Swedish multi-centre study comparing real-time polymerase chain reaction and multicolour flow cytometry. 08456.x

9. Kerst $\mathrm{G}$, Kreyenberg $\mathrm{H}$, Roth $\mathrm{C}$, et al. Concurrent detection of minimal residual disease (MRD) in childhood acute lymphoblastic leukaemia by flow cytometry and real-time PCR. Br J Haematol. 2005;128(6):774-782. https://doi.org/10. and real-time PCR. Br J Haemato

10. Dai $Q$, Liu $X$, Yang $H$, et al. No prognostic significance of immunophenotypic changes at the end of remission induction therapy in children with B-lineage acute lymphoblastic leukemia. Leuk Res. 2018;68(March):57-61. https://doi.org/10 lymphoblastic leukemia. Le

11. Wood BL. Principles of minimal residual disease detection for hematopoietic neoplasms by flow cytometry. Cytom Part B - Clin Cytom. 2016;90(1):47-53. https://doi.org/10.1002/cyto.b.21239

12. Sepdek L, Bulsa J, Sonsala A, et al. The immunophenotypes of blast cells in B-cell precursor acute lymphoblastic leukemia: How different are they from their normal counterparts? Cytom Part B - Clin Cytom. 2014;86(5):329-339. https:// doi.org/10.1002/cyto.b.21176

13. Theunissen $P$, Mejstrikova $E$, Sedek L, et al. Standardized flow cytometry for highly sensitive MRD measurements in B-cell acute lymphoblastic leukemia. Blood. 2017;129(3):347-358. https://doi.org/10.1182/blood-2016-07-726307

14. Borowitz MJ, Devidas M, Hunger SP, et al. Clinical significance of minimal residual disease in childhood acute lymphoblastic leukemia and its relationship to other prognostic factors: A Children's Oncology Group study. Blood. 2008;111(12): 5477-5485. https://doi.org/10.1182/blood-2008-01-132837

15. Theunissen PMJ, Sedek L, De Haas V, et al. Detailed immunophenotyping of B-cel precursors in regenerating bone marrow of acute lymphoblastic leukaemia patients: Implications for minimal residual disease detection. $\mathrm{Br} J$ Haematol. 2017;178(2):257-266. https://doi.org/10.1111/bjh.14682

16. Farahat N, Morilla $A$, Owusu-Ankomah $\mathrm{K}$, et al. Detection of minimal residual disease in B-lineage acute lymphoblastic leukaemia by quantitative flow cytometry. $\mathrm{Br} J$ Haematol [serial on the Internet]. [cited Nov 2018] 1998;101(1):158-164. Available from: http://www.ncbi.nlm.nih.gov/pubmed/9576196.

17. Gupta S, Devidas M, Loh ML, et al. Flow cytometric vs morphologic assessment of remission in childhood acute lymphoblastic leukemia : A report from the children's oncology. Leukemia. 2018;32(6):1370-1379. https://doi.org/10.1038/s41375 018-0039-7
18. Mayosi BM, Flisher AJ, Lalloo UG, et al. Health in South Africa 4 The burden of non-communicable diseases in South Africa. Lancet. 2009:374(9693):934-947. http://doi.org/10.1016/S0140-6736(09)61087-4

19. Green T, Bron D, Chomienne C, et al. Costs of haematological disease high and rising. Lancet Haematol. 2019;3:353-354. https://doi.org/10.1016/S2352-3026(16)30074-6

20. Kalina T, Flores-Montero J, Van Der Velden VHJ, et al. EuroFlow standardization of flow cytometer instrument settings and immunophenotyping protocols. 2012;26(9):1986-2010. https://doi.org/10.1038/leu.2012.122

21. Van Dongen JJM, Lhermitte L, Bottcher $S$, et al. EuroFlow antibody panels for standardized $\mathrm{n}$-dimensional flow cytometric immunophenotyping of normal, reactive and malignant leukocytes. 2012;26(9):1908-1975.

22. Riehm H, Schrappe M, Reiter A. Treatment protocol ALL-BFM 95 for children and adolescents with acute lymphoblastic leukemia: A cooperative multicenter trial of the German Society for Pediatric Hematol Oncol. 1998;1:8-80.

23. Vora A, Goulden N, Mitchell C, et al. Augmented post-remission therapy for a minimal residual disease-defined high-risk subgroup of children and young people with clinical standard-risk and intermediate-risk acute lymphoblastic leukaemia (UKALL 2003): A randomised controlled trial. Lancet Oncol. 2014;15(8):809-818. http://doi.org/10.1016/S1470-2045(14)70243-8

24. Gaipa G, Basso G, Biondi A, Campana D. Detection of minimal residual disease in pediatric acute lymphoblastic leukemia. Cytom Part B - Clin Cytom. pediatric acute lymphoblastic leukemia. Cytom Part B -

25. Athale UH, Frcp C, Gibson PJ, et al. Minimal residual disease and childhood leukemia : Standard of care recommendations from the pediatric oncology group of Ontario MRD Working Group the principle of risk stratification of treatment. Pediatr Blood Cancer. 2016;63(6):973-982. https://doi.org/10.1002/pbc.25939

26. Pieters R, De Groot-Kruseman H, Van Der Velden V, et al. Successful therapy reduction and intensification for childhood acute lymphoblastic leukemia based on minimal residual disease monitoring: Study ALL10 from the Dutch Childhood Oncology Group. J Clin Oncol. 2016;34(22):2591-2601.

27. Campana D, Pui C. Evidence-based focused review minimal residual disease Guided therapy in childhood acute lymphoblastic leukemia Case presentations. Blood. 2017;129(14):1913-1919. https://doi.org/10.1182/blood-2016-12-725804

28. Szczepański T. Why and how to quantify minimal residual disease in acute lymphoblastic leukemia? Leukemia. 2007;21(4):622-626. https://doi.org/10. 1038/sj.leu.2404603

29. Dworzak MN, Buldini B, Gaipa G, et al. AIEOP-BFM consensus guidelines 2016 for flow cytometric immunophenotyping of pediatric acute lymphoblastic leukemia. 2018;93(1):82-93. https://doi.org/10.1002/cyto.b.21518

30. Borowitz MJ, Devidas M, Hunger SP, et al. Clinical significance of minimal residual disease in childhood acute lymphoblastic leukemia and its relationship to other prognostic factors: A Children's Oncology Group study. Blood. 2019;111(12): 5477-5486. https://doi.org/10.1182/blood-2008-01-132837

31. Borowitz MJ, Pullen DJ, Shuster JJ, et al. Minimal residual disease detection in childhood precursor-B-cell acute lymphoblastic leukemia: Relation to other risk
factors. A Children's Oncology Group study. Leukemia. 2003;17(8):1566-1572. factors. A Children's Oncology Group stur
https://doi.org/10.1038/sj.leu.2403001

32. Coustan-Smith E, Sancho J, Hancock ML, et al. Clinical importance of minimal residual disease in childhood acute lymphoblastic leukemia. Blood. 2015;96(8): residual dis $2691-2697$.

33. Chen X, Wood BL. How do we measure MRD in ALL and how should measurements affect decisions. Re: Treatment and prognosis? Best Pract Res Clin Haematol. 2017;30(3):237-248. https://doi.org/10.1016/j.beha.2017.07.002

34. Bao P, Zheng Y, Wang C, Gu K, Jin F, Lu W. Time trends and characteristics of childhood cancer among children age $0-14$ in Shanghai. Pediatr Blood Cancer. 2009;53(1):13-16. https://doi.org/10.1002/pbc.21939

35. Salari F, Shahjahani M. Minimal residual disease in acute lymphoblastic leukemia: Optimal methods and clinical relevance, pitfalls and recent approaches. Med Oncol. 2014;31(266):1-9. https://doi.org/10.1007/s12032-014-0266-3

36. Sekiya $Y, X u Y$, Muramatsu $H$, et al. Clinical utility of next-generation sequencingbased minimal residual disease in paediatric B-cell acute lymphoblastic leukaemia. Br J Haematol. 2017;176(2):248-257. https://doi.org/10.1111/bjh.14420

37. Fossat $\mathrm{C}$, Roussel M, Arnoux I, et al. Methodological aspects of minimal residual disease assessment by flow cytometry in acute lymphoblastic leukemia: A French multicenter study. Cytom Part B - Clin Cytom. 2015;88(1):21-29. https://doi. org/10.1002/cyto.b.21195

38. Shaver AC, Seegmiller AC. B lymphoblastic leukemia minimal residual disease assessment by flow cytometric analysis. Clin Lab Med. 2017;37(4):771-785. https://doi.org/10.1016/j.cll.2017.07.005

39. Gazzola A, Mannu C, Rossi M, et al. The evolution of clonality testing in the diagnosis and monitoring of hematological malignancies. 2014;5(2):35-47. https://doi.org/10.1177/2040620713519729

40. Karawajew L, Dworzak $M$, Ratei $R$, et al. Minimal residual disease analysis by eight-color flow cytometry in relapsed childhood acute lymphoblastic leukemia. Haematologica. 2015;100(7):935-944. https://doi.org/10.3324/haematol.2014. 116707

41. Gray A, Vawda Y. Health legislation and policy. In: Rispel LC, Padarath AE, editors South African health review 2018. Durban: Health Systems Trust, 2018. 